\title{
New Lax Pairs and Darboux Transformation and Its Application to a Shallow Water Wave Model of Generalized KdV Type
}

\author{
Huizhang Yang ${ }^{1}$ and Weiguo Rui ${ }^{2}$ \\ ${ }^{1}$ College of Mathematics of Honghe University, Mengzi, Yunnan 661100, China \\ ${ }^{2}$ College of Mathematics of Chongqing Normal University, Chongqing 401331, China
}

Correspondence should be addressed to Weiguo Rui; weiguorhhu@aliyun.com

Received 30 March 2013; Accepted 5 September 2013

Academic Editor: Sarp Adali

Copyright (c) $2013 \mathrm{H}$. Yang and W. Rui. This is an open access article distributed under the Creative Commons Attribution License, which permits unrestricted use, distribution, and reproduction in any medium, provided the original work is properly cited.

\begin{abstract}
New Lax pairs of a shallow water wave model of generalized KdV equation type are presented. According to this Lax pair, we constructed a new spectral problem. By using this spectral problem, we constructed Darboux transformation with the help of a gauge transformation. Applying this Darboux transformation, some new exact solutions including double-soliton solution of the shallow water wave model of generalized $\mathrm{KdV}$ equation type are obtained. In order to visually show dynamical behaviors of these double soliton solutions, we plot graphs of profiles of them and discuss their dynamical properties.
\end{abstract}

\section{Introduction}

It is well known that the Lax pair and Darboux transformation can be employed to obtain multisoliton solution of nonlinear evolution equations. Darboux transformations provide us with purely algebraic, powerful method to construct solutions for systems of nonlinear equations. In recent years, more and more researchers used the Lax pair and Darboux transformation to investigate soliton solutions of classical nonlinear wave equations and some new soliton equations which were generated by new spectral problems; see [1$30]$ and references cited therein. In general, a systematical theory on such Darboux transformation even for $n \times n$ matrix spectral problem and the resulting zero curvature equation has a beautiful algebraic structure for associated evolution equations, which tells symmetry algebras of the obtained evolution equations; see $[31,32]$ and references cited therein. Sometimes, it is found that there are many infinity symmetries from the adopted zero curvature equation.

In this paper, we will investigate the Lax pairs, Darboux transformation, and double soliton solutions of the following famous shallow water wave model of generalized KdV equation type:

$$
u_{t}-\frac{h_{0}^{2}}{3} u_{x x t}+c_{0} u_{x}+\frac{3 \alpha}{2} u u_{x}-\frac{c_{0} h_{0}^{2}}{6} u_{x x x}
$$

$$
=\frac{\alpha h_{0}^{2}}{3} u_{x} u_{x x}+\frac{\alpha h_{0}^{2}}{6} u u_{x x x}
$$

which appeared in [33], where $0<\alpha \ll 1$ is a smallamplitude parameter. Only dropping the right-hand side of (1) gives BBM equation. Dropping the right-hand side of (1) and replacing the term $u_{x x t}$ by the term $-c_{0} u_{x x x}$ give KdV equation [34]. Thus, (1) can be seen as a BBM equation extended by retaining higher order terms in an asymptotic expansion in terms of the small-amplitude parameter $\alpha$. Dropping the term $-\left(c_{0} h_{0}^{2} / 6\right) u_{x x x}$ from (1) and letting $h_{0}=$ $\sqrt{3}, c_{0}=2 \kappa, \alpha=2$, (1) becomes the celebrated CamassaHolm equation [33] as follows:

$$
u_{t}+2 \kappa-u_{x x t}+3 u u_{x}=2 u_{x} u_{x x}+u u_{x x x}
$$

where $u$ is the fluid velocity in the $x$ direction (or equivalent to the height of the water's free surface above a flat bottom), $\kappa$ is a constant related to the critical shallow water wave speed, and subscripts denote partial derivatives. Letting $c_{0}=1, v=$ $-(1 / 3) h_{0}^{2}, \gamma=(3 / 2) \alpha, \beta=-(1 / 6) h_{0}^{2} c_{0}$, (1) can be rewritten as $u_{t}+u_{x}+v u_{x x t}+\beta u_{x x x}+\gamma u u_{x}+\frac{1}{3} \gamma \nu\left(u u_{x x x}+2 u_{x} u_{x x}\right)=0$, 
which comes from physical considerations via the methodology introduced by Fuchssteiner and Fokas in $[35,36]$. The Lax pairs of (3) with $\gamma=1$ are given by $[37,38]$ as follows:

$$
\begin{gathered}
\Psi_{x x}=\left[k^{2}(1-v)+\frac{v}{4 v}\right] \Psi=0, \quad \sigma v=u+v u_{x x}, \\
\sigma=\frac{\beta-v}{2 v}, \\
\Psi_{t}+\left(-\frac{1}{2}+c\right) \Psi+\left(u+\frac{\beta}{v}+\frac{2 \sigma}{4 v k^{2}-1}\right) \Psi_{x}=0,
\end{gathered}
$$

where $c$ is an arbitrary constant. It is a pity that the [37] is not a formal publication, and it is only preprint paper, so we cannot know its reality contents whether the authors have obtained soliton solutions of (3) by the Lax pairs (4) and (5). In fact, (3) has been studied by many authors in recent years; see the following brief introductions.

In [39], by using the bifurcation theory of dynamic system, some subsection-function and implicit function solutions such as compactons, solitary waves, smooth periodic waves, and nonsmooth periodic waves with peaks as well as the existence conditions have been presented by Bi. By using the same method, Li and Zhang [40] studied a generalization form of the modified $\mathrm{KdV}$ equation, which is more complex than (3). In [40], the existence of solitary wave, kink and antikink wave solutions, and uncountably many smooth and nonsmooth periodic wave solutions are discussed. By using the improved method named integral bifurcation method [41], Rui et al. [42] obtain all kinds of soliton-like or kink-like wave solutions, periodic wave solutions with loop or without loop, smooth compacton-like periodic wave solutions, and nonsmooth periodic cusp wave solutions for (3). In [43], Long and Chen discussed the existence of solitary wave, cusp wave, periodic wave, periodic cusp wave, and compactons were for (3). From the above references, (1) (i.e., (3)) is a very important water wave model.

The rest of this paper is organized as follows. In Section 2, we will derive new Lax pair and Darboux transformation of (1). In Section 3, by using this Darboux transformation, we will investigate soliton solutions of (1) and discuss the dynamic properties of these soliton solutions.

\section{Lax Pair and Darboux Transformation of (1)}

Through a series of tedious computation, we obtain Lax pairs of (1) as follows:

$$
\begin{gathered}
\phi_{x x}=\left(-\frac{c_{0}}{4 \lambda \alpha}+\frac{3}{4 h_{0}^{2}}-\frac{u-(1 / 3) h_{0}^{2} u_{x x}}{2 \lambda}\right) \phi, \\
\phi_{t}=-\left(\frac{c_{0}}{2}+\frac{3 \lambda \alpha}{2 h_{0}^{2}}+\frac{\alpha}{2} u\right) \phi_{x}+\frac{\alpha}{4} u_{x} \phi
\end{gathered}
$$

Obviously, the Lax pairs (6) and (7) are different from the Lax pairs (4) and (5) under $c_{0}=1, v=-(1 / 3) h_{0}^{2}, \gamma=$ $(3 / 2) \alpha, \beta=-(1 / 6) h_{0}^{2} c_{0}$. They are new Lax pairs which we obtained. By using the new Lax pairs (6) and (7), we will construct a Darboux transformation for obtaining soliton solutions of (1).

First, we consider the following spectral problems:

$$
\phi_{x}=\mathbf{M} \phi, \quad \phi_{t}=\mathbf{N} \phi,
$$

with

$$
\begin{gathered}
\mathbf{M}=\left(\begin{array}{cc}
0 & c_{0} \\
-\frac{3}{4 \lambda \alpha}+\frac{3}{4 h_{0}^{2}}-\frac{u-(1 / 3) h_{0}^{2} u_{x x}}{2 \lambda} & 0
\end{array}\right), \\
\frac{\alpha}{4} u_{x}=\left(\begin{array}{c}
\left.\frac{c_{0}}{2}+\frac{3 \lambda \alpha}{2 h_{0}^{2}}+\frac{\alpha}{2} u\right) \\
\frac{\alpha}{4} u_{x x}-\left(\frac{c_{0}}{2}+\frac{3 \lambda \alpha}{2 h_{0}^{2}}+\frac{\alpha}{2} u\right)\left(-\frac{c_{0}}{4 \lambda \alpha}+\frac{3}{4 h_{0}^{2}}-\frac{u-(1 / 3) h_{0}^{2} u_{x x}}{2 \lambda}\right)
\end{array}\right),
\end{gathered}
$$

where $\alpha$ is a constant, $\lambda$ is a spectral parameter, and $u$ is a potential function. From compatibility, condition $\phi_{x x t}=\phi_{t x x}$ yields a zero curvature equation $\mathbf{M}_{t}-\mathbf{N}_{x}+[\mathbf{M}, \mathbf{N}]=\mathbf{O}$. Substituting $\mathbf{M}, \mathbf{N}$ into the zero curvature equation, by a direct calculation, (1) is obtained successfully.

Next, we will construct a Darboux Transformation (DT) of the spectral problems (8). In fact, the DT is actually a gauge transformation of the spectral problems (8). It is required that $\bar{\phi}$ also satisfies the same form of spectral problems

$$
\begin{aligned}
\bar{\phi}_{x}=\overline{\mathbf{M}} \bar{\phi}, & \overline{\mathbf{M}}=\left(\mathbf{T}_{x}+\mathbf{T M}\right) \mathbf{T}^{-1}, \\
\bar{\phi}_{t}=\overline{\mathbf{N}} \bar{\phi}, & \overline{\mathbf{N}}=\left(\mathbf{T}_{t}+\mathbf{T N}\right) \mathbf{T}^{-1} .
\end{aligned}
$$

It means that we have to find a matrix $\mathbf{T}$ such that the old potential $u$ is replaced by the new one $\bar{u}$.

Suppose

$$
\mathbf{T}=\mathbf{T}(\lambda)=\left(\begin{array}{ll}
A(\lambda) & B(\lambda) \\
C(\lambda) & D(\lambda)
\end{array}\right)
$$


where

$$
\begin{gathered}
A(\lambda)=A_{N}\left(\lambda^{N}+\sum_{k=0}^{N-1} A_{k} \lambda^{k}\right), \\
B(\lambda)=A_{N}\left(\sum_{k=0}^{N-1} B_{k} \lambda^{k}\right), \\
C(\lambda)=\frac{1}{A_{N}}\left(\sum_{k=0}^{N-1} C_{k} \lambda^{k}\right), \\
D(\lambda)=\frac{1}{A_{N}}\left(\lambda^{N}+\sum_{k=0}^{N-1} D_{k} \lambda^{k}\right),
\end{gathered}
$$

and $A_{N}, A_{k}, B_{k}, C_{k}$, and $D_{k}(0 \leq k \leq N-1)$ are functions of $x$ and $t$.

Let $\phi\left(\lambda_{j}\right)=\left(\phi_{1}\left(\lambda_{j}\right), \phi_{2}\left(\lambda_{j}\right)\right)^{T}, \psi\left(\lambda_{j}\right)=\left(\psi_{1}\left(\lambda_{j}\right), \psi_{2}\left(\lambda_{j}\right)\right)^{T}$ be two basic solutions of (8). From (10), there exist constants $r_{j}(0 \leq j \leq N-1)$, which satisfy

$$
\begin{aligned}
& \left(A\left(\lambda_{j}\right) \phi_{1}\left(\lambda_{j}\right)+B\left(\lambda_{j}\right) \phi_{2}\left(\lambda_{j}\right)\right) \\
& \quad-r_{j}\left(A\left(\lambda_{j}\right) \psi_{1}\left(\lambda_{j}\right)+B\left(\lambda_{j}\right) \psi_{2}\left(\lambda_{j}\right)\right)=0, \\
& \left(C\left(\lambda_{j}\right) \phi_{1}\left(\lambda_{j}\right)+D\left(\lambda_{j}\right) \phi_{2}\left(\lambda_{j}\right)\right) \\
& \quad-r_{j}\left(C\left(\lambda_{j}\right) \psi_{1}\left(\lambda_{j}\right)+D\left(\lambda_{j}\right) \psi_{2}\left(\lambda_{j}\right)\right)=0 .
\end{aligned}
$$

Further, (16) can be written as a linear algebraic system

$$
A\left(\lambda_{j}\right)+\delta_{j} B\left(\lambda_{j}\right)=0, \quad C\left(\lambda_{j}\right)+\delta_{j} D\left(\lambda_{j}\right)=0 .
$$

That is

$$
\sum_{k=0}^{N-1}\left(A_{k}+\delta_{j} B_{k}\right) \lambda_{j}^{k}=-\lambda_{j}^{N}, \quad \sum_{k=0}^{N-1}\left(C_{k}+\delta_{j} D_{k}\right) \lambda_{j}^{k}=-\delta_{j} \lambda_{j}^{N},
$$

where

$$
\delta_{j}=\frac{\phi_{2}\left(\lambda_{j}\right)-r_{j} \psi_{2}\left(\lambda_{j}\right)}{\phi_{1}\left(\lambda_{j}\right)-r_{j} \psi_{1}\left(\lambda_{j}\right)}, \quad 1 \leq j \leq 2 N,
$$

and the constants $\lambda_{j}\left(\lambda_{k} \neq \lambda_{s}\right.$ as $\left.k \neq s\right), r_{j}$ are suitably chosen such that determinant of coefficients for (18) is nonzero. Therefore, $A_{N}, A_{k}, B_{k}, C_{k}$, and $D_{k}(0 \leq k \leq N-1)$ are uniquely determined by (18).

Equations (14) and (15) show that the $\operatorname{det} \mathbf{T}(\lambda)$ is a $2 N$ thorder polynomial in $\lambda$, and

$$
\operatorname{det} \mathbf{T}\left(\lambda_{j}\right)=A\left(\lambda_{j}\right) D\left(\lambda_{j}\right)-B\left(\lambda_{j}\right) C\left(\lambda_{j}\right) .
$$

On the other hand, from (17), we have $A\left(\lambda_{j}\right)=-\delta_{j} B\left(\lambda_{j}\right)$, $C\left(\lambda_{j}\right)=-\delta_{j} D\left(\lambda_{j}\right)$. Thus we have

$$
\operatorname{det} \mathbf{T}(\lambda)=\beta \prod_{j=1}^{2 N-1}\left(\lambda-\lambda_{j}\right),
$$

where $\beta$ is independent of $\lambda$. Equation (21) implies that $\lambda_{j}(1 \leq j \leq 2 N)$ are $2 N$ roots of $\operatorname{det} \mathbf{T}(\lambda)$.

Second, we prove the following theory of Darboux transformation for special variable.

Theorem 1. Let $A_{N}$ satisfy

$$
A_{N}^{2}=1 .
$$

Then the matrix $\overline{\mathbf{M}}$ determined by (11) has the same form as $\mathbf{M}$; that is,

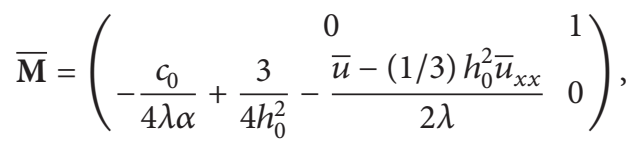

where the transformation from the old potential $u$ into new one $\bar{u}$ is given by

$$
\begin{gathered}
\bar{u}=u+\frac{6}{h_{0}^{2}} A_{N-1}, \\
A_{N-1, x}=C_{N-1}-\frac{3}{4 h_{0}^{2}} B_{N-1}, \\
B_{N-1, x}=D_{N-1}-A_{N-1}, \\
C_{N-1, x}=\frac{3}{4 h_{0}^{2}}\left(A_{N-1}-D_{N-1}\right)+\frac{c_{0}}{4 \alpha}+\frac{u-(1 / 3) h_{0}^{2} u_{x x}}{2}, \\
D_{N-1, x}=\frac{3}{4 h_{0}^{2}} B_{N-1}-C_{N-1} .
\end{gathered}
$$

Proof. Let $\mathbf{T}^{-1}=\mathbf{T}^{*} / \operatorname{det} \mathbf{T}$ and

$$
\left(\mathbf{T}_{x}+\mathbf{T M}\right) \mathbf{T}^{*}=\left(\begin{array}{ll}
f_{11}(\lambda) & f_{12}(\lambda) \\
f_{21}(\lambda) & f_{22}(\lambda)
\end{array}\right) .
$$

It is easy to see that $f_{11}(\lambda)$ and $f_{22}(\lambda)$ are $2 N$ th-order polynomials in $\lambda, f_{12}(\lambda)$ and $f_{21}(\lambda)$ are $(2 N-1)$ th-order polynomials in $\lambda$. From (19) and (8), we find

$$
\delta_{j x}=-\frac{c_{0}}{4 \lambda \alpha}+\frac{3}{4 h_{0}^{2}}-\frac{u-(1 / 3) h_{0}^{2} u_{x x}}{2 \lambda}-\delta_{j}^{2} .
$$

Through direct calculation, all $\lambda_{j}(0 \leq j \leq 2 N)$ are roots of $f_{n s}(n, s=1,2)$. Together with (21) and (26), we get

$$
\left(\mathbf{T}_{x}+\mathbf{T M}\right) \mathbf{T}^{*}=(\operatorname{det} \mathbf{T}) P(\lambda),
$$

with

$$
P(\lambda)=\left(\begin{array}{ll}
p_{11}^{(0)} & p_{12}^{(0)} \\
p_{21}^{(0)} & p_{22}^{(0)}
\end{array}\right)
$$

where $p_{n s}^{(0)}(n, s=1,2)$ are independent of spectral parameter $\lambda$. Indeed, (28) can be written as

$$
\mathbf{T}_{x}+\mathbf{T M}=P(\lambda) \mathbf{T} .
$$


Comparing the coefficients of $\lambda^{N}$ in (30), we find

$$
\begin{gathered}
p_{11}^{(0)}=-p_{22}^{(0)}=\partial_{x} \ln A_{N}, \quad p_{12}^{(0)}=A_{N}^{2}, \\
p_{21}^{(0)}=\frac{1}{A_{N}^{2}} \frac{3}{4 h_{0}^{2}} .
\end{gathered}
$$

Substituting (22) into (31) yields

$$
p_{11}^{(0)}=-p_{22}^{(0)}=0, \quad p_{12}^{(0)}=A_{N}^{2}=1 .
$$

From (22), (24), (25), and (31) and noticing $\bar{u}$ in (23), we get

$$
p_{21}^{(0)}=-\frac{c_{0}}{4 \lambda \alpha}+\frac{3}{4 h_{0}^{2}}-\frac{\bar{u}-(1 / 3) h_{0}^{2} \bar{u}_{x x}}{2 \lambda} .
$$

Thus $P(\lambda)=\overline{\mathbf{M}}$. The proof of Theorem 1 is completed.

Finally, by using same way to Theorem 1, we prove that $\overline{\mathbf{N}}$ in (12) has the same form as $\mathbf{N}$ under the transformation (10) and (24); see the following theory and its proof.

Theorem 2. The matrix $\overline{\mathbf{N}}$ defined by (12) has the same type as $\mathbf{N}$, in which the old potential $u$ is mapped into $\bar{u}$ via the same DT (24).

Proof. Let $\mathbf{T}^{-1}=\mathbf{T}^{*} / \operatorname{det} \mathbf{T}$ and

$$
\left(\mathbf{T}_{t}+\mathbf{T N}\right) \mathbf{T}^{*}=\left(\begin{array}{ll}
g_{11}(\lambda) & g_{12}(\lambda) \\
g_{21}(\lambda) & g_{22}(\lambda)
\end{array}\right)
$$

It is easy to see that $g_{11}(\lambda)$ and $g_{22}(\lambda)$ are $2 N$ th-order polynomials in $\lambda, g_{12}(\lambda)$ and $g_{21}(\lambda)$ are $(2 N+1)$ th-order polynomials in $\lambda$. By using (19) and (8), we obtain

$$
\begin{aligned}
\delta_{j t}= & \frac{\alpha}{4} u_{x x}-\left(\frac{c_{0}}{2}+\frac{3 \lambda \alpha}{2 h_{0}^{2}}+\frac{\alpha}{2} u\right) \\
& \times\left(-\frac{c_{0}}{4 \lambda \alpha}+\frac{3}{4 h_{0}^{2}}-\frac{u-(1 / 3) h_{0}^{2} u_{x x}}{2 \lambda}\right) \\
& -\frac{\alpha}{2} u_{x} \delta_{j}+\left(\frac{c_{0}}{2}+\frac{3 \lambda \alpha}{2 h_{0}^{2}}+\frac{\alpha}{2} u\right) \delta_{j}^{2} .
\end{aligned}
$$

Through direct calculation, all $\lambda_{j}(0 \leq j \leq 2 N)$ are roots of $g_{n s}(n, s=1,2)$. Together with (21) and (34), we get

$$
\left(\mathbf{T}_{t}+\mathbf{T N}\right) \mathbf{T}^{*}=(\operatorname{det} \mathbf{T}) Q(\lambda)
$$

with

$$
Q(\lambda)=\left(\begin{array}{cc}
q_{11}^{(0)} & q_{12}^{(1)} \lambda+q_{12}^{(0)} \\
q_{21}^{(1)} \lambda+q_{21}^{(0)} & q_{22}^{(0)}
\end{array}\right)
$$

where $q_{n s}^{(l)}(n, s=1,2, l=0,1)$ are independent of spectral parameter $\lambda$. Equation (37) can be written as

$$
\mathbf{T}_{t}+\mathbf{T N}=Q(\lambda) \mathbf{T}
$$

Comparing the coefficients of $\lambda^{N+1}$ and $\lambda^{N}$ in (38) leads to

$$
\begin{gathered}
q_{12}^{(1)}=-\frac{3 \alpha}{2 h_{0}^{2}} A_{N}^{2}, \quad q_{21}^{(1)}=-\frac{1}{A_{N}^{2}} \frac{3 \alpha}{2 h_{0}^{2}} \frac{3}{4 h_{0}^{2}}, \\
q_{11}^{(0)}=\partial_{t} \ln A_{N}+\frac{\alpha}{4} u_{x}-\frac{3 \alpha}{2 h_{0}^{2}} \frac{3}{4 h_{0}^{2}} B_{N-1}+\frac{3 \alpha}{2 h_{0}^{2}} C_{N-1}, \quad(40) \\
q_{12}^{(0)}=-\left(\frac{c_{0}}{2}+\frac{\alpha}{2} u\right) A_{N}^{2}-\frac{3 \alpha}{2 h_{0}^{2}} A_{N}^{2} A_{N-1}+\frac{3 \alpha}{2 h_{0}^{2}} A_{N}^{2} D_{N-1}, \\
q_{21}^{(0)}=\frac{1}{A_{N}^{2}} \frac{\alpha}{4} u_{x x}-\frac{1}{A_{N}^{2}} \frac{3}{4 h_{0}^{2}}\left(\frac{c_{0}}{2}+\frac{\alpha}{2} u\right)-\frac{1}{A_{N}^{2}} \frac{3 \alpha}{2 h_{0}^{2}} \frac{3}{4 h_{0}^{2}} D_{N-1} \\
+\frac{1}{A_{N}^{2}} \frac{3 \alpha}{2 h_{0}^{2}}\left(\frac{c_{0}}{4 \alpha}+\frac{u-(1 / 3) h_{0}^{2} u_{x x}}{2}\right) \\
+\frac{A_{N-1}}{A_{N}^{2}} \frac{3 \alpha}{2 h_{0}^{2}} \frac{3}{4 h_{0}^{2}},
\end{gathered}
$$

$$
q_{22}^{(0)}=-\partial_{t} \ln A_{N}-\frac{3 \alpha}{2 h_{0}^{2}} C_{N-1}-\frac{\alpha}{4} u_{x}+\frac{3 \alpha}{2 h_{0}^{2}} \frac{3}{4 h_{0}^{2}} B_{N-1} .
$$

Substituting (22), (24), and (25) into (40) to (43), we can get

$$
\begin{aligned}
q_{11}^{(0)} & =\frac{\alpha}{4} u_{x}-\frac{3 \alpha}{2 h_{0}^{2}} \frac{3}{4 h_{0}^{2}} B_{N-1}+\frac{3 \alpha}{2 h_{0}^{2}} C_{N-1} \\
& =\frac{\alpha}{4} u_{x}-\frac{3 \alpha}{2 h_{0}^{2}} A_{N-1, x} \\
& =\frac{\alpha}{4} \bar{u}_{x}, \\
q_{12}^{(1)} \lambda & +q_{12}^{(0)} \\
& =-\frac{3 \alpha}{2 h_{0}^{2}} \lambda-\left(\frac{c_{0}}{2}+\frac{\alpha}{2} u\right)-\frac{3 \alpha}{2 h_{0}^{2}} A_{N-1}+\frac{3 \alpha}{2 h_{0}^{2}} D_{N-1} \\
& =-\frac{3 \alpha}{2 h_{0}^{2}} \lambda-\left(\frac{c_{0}}{2}+\frac{\alpha}{2} u\right)-\frac{3 \alpha}{h_{0}^{2}}+\frac{3 \alpha}{2 h_{0}^{2}}\left(A_{N-1}+D_{N-1}\right) \\
& =-\left(\frac{c_{0}}{2}+\frac{3 \lambda \alpha}{2 h_{0}^{2}}+\frac{\alpha}{2} \bar{u}\right), \\
q_{22}^{(0)} & =-\frac{3 \alpha}{2 h_{0}^{2}} C_{N-1}-\frac{\alpha}{4} u_{x}+\frac{3 \alpha}{2 h_{0}^{2}} \frac{3}{4 h_{0}^{2}} B_{N-1} \\
& =-\frac{\alpha}{4} \bar{u}_{x}, \\
& \\
&
\end{aligned}
$$




$$
\begin{aligned}
q_{21}^{(1)} \lambda+ & q_{21}^{(0)} \\
= & -\frac{3 \alpha}{2 h_{0}^{2}} \frac{3}{4 h_{0}^{2}} \lambda+\frac{\alpha}{4} u_{x x}-\frac{3}{4 h_{0}^{2}}\left(\frac{c_{0}}{2}+\frac{\alpha}{2} u\right)-\frac{3 \alpha}{2 h_{0}^{2}} \frac{3}{4 h_{0}^{2}} D_{N-1} \\
& +\frac{3 \alpha}{2 h_{0}^{2}}\left(\frac{c_{0}}{4 \alpha}+\frac{u-(1 / 3) h_{0}^{2} u_{x x}}{2}\right)+\frac{3 \alpha}{2 h_{0}^{2}} \frac{3}{4 h_{0}^{2}} A_{N-1} \\
= & \frac{\alpha}{4} u_{x x}-\frac{3}{4 h_{0}^{2}}\left(\frac{c_{0}}{2}+\frac{3 \lambda \alpha}{2 h_{0}^{2}}+\frac{\alpha}{2} u\right)+\frac{3 \alpha}{2 h_{0}^{2}} C_{N-1, x} .
\end{aligned}
$$

On the other hand, from (24), (25), and (33), we have

$$
\begin{aligned}
\frac{\alpha}{4} \bar{u}_{x x}-\left(\frac{c_{0}}{2}+\frac{3 \lambda \alpha}{2 h_{0}^{2}}+\frac{\alpha}{2} \bar{u}\right) \\
\quad \times\left(-\frac{c_{0}}{4 \lambda \alpha}+\frac{3}{4 h_{0}^{2}}-\frac{\bar{u}-(1 / 3) h_{0}^{2} \bar{u}_{x x}}{2 \lambda}\right) \\
=\frac{\alpha}{4} \bar{u}_{x x}-\left(\frac{c_{0}}{2}+\frac{3 \lambda \alpha}{2 h_{0}^{2}}+\frac{\alpha}{2} \bar{u}\right) \frac{3}{4 h_{0}^{2}} \\
=\frac{\alpha}{4} u_{x x}+\frac{3 \alpha}{2 h_{0}^{2}} A_{N-1, x x} \\
\quad-\left(\frac{c_{0}}{2}+\frac{3 \lambda \alpha}{2 h_{0}^{2}}+\frac{\alpha}{2} u\right) \frac{3}{4 h_{0}^{2}}-\frac{6 \alpha}{2 h_{0}^{2}} \frac{3}{4 h_{0}^{2}} A_{N-1} \\
=\frac{\alpha}{4} u_{x x}-\left(\frac{c_{0}}{2}+\frac{3 \lambda \alpha}{2 h_{0}^{2}}+\frac{\alpha}{2} u\right) \frac{3}{4 h_{0}^{2}}+\frac{3 \alpha}{2 h_{0}^{2}} C_{N-1, x} .
\end{aligned}
$$

From (44) and (45), obviously we have

$$
\begin{aligned}
q_{21}^{(1)} \lambda+q_{21}^{(0)}= & \frac{\alpha}{4} \bar{u}_{x x}-\left(\frac{c_{0}}{2}+\frac{3 \lambda \alpha}{2 h_{0}^{2}}+\frac{\alpha}{2} \bar{u}\right) \\
& \times\left(-\frac{c_{0}}{4 \lambda \alpha}+\frac{3}{4 h_{0}^{2}}-\frac{\bar{u}-(1 / 3) h_{0}^{2} \bar{u}_{x x}}{2 \lambda}\right) .
\end{aligned}
$$

\section{Exact Soliton Solution of (1) and Its Dynamic Properties}

In this section, we will construct the explicit solutions of the integrable shallow water wave (1) by using the Darboux transformation (24)

Choosing $u=u_{0}\left(u_{0}\right.$ is an arbitrary constant) as a seed solution of (1), and substituting $u=u_{0}$ into the spectral problems (8). Then we get two basic solutions of (8) as follows:

$$
\begin{gathered}
\phi\left(\lambda_{j}\right)=\left(\sqrt{-\frac{c_{0}}{4 \lambda_{j} \alpha}+\frac{3}{4 h_{0}^{2}}-\frac{u_{0}}{2 \lambda_{j}}} \sinh \mu_{j}\right), \\
\psi\left(\lambda_{j}\right)=\left(\sqrt{-\frac{c_{0}}{4 \lambda_{j} \alpha}+\frac{3}{4 h_{0}^{2}}-\frac{u_{0}}{2 \lambda_{j}}} \cosh \mu_{j}\right)
\end{gathered}
$$

with

$$
\mu_{j}=\sqrt{-\frac{c_{0}}{4 \lambda_{j} \alpha}+\frac{3}{4 h_{0}^{2}}-\frac{u_{0}}{2 \lambda_{j}}}\left[x-\left(\frac{c_{0}}{2}+\frac{3 \lambda_{j} \alpha}{2 h_{0}^{2}}+\frac{u_{0} \alpha}{2}\right) t\right],
$$

where $\lambda_{j}$ is a nonzero constant and $0<j \leq 2 N$.

According to (19), we have

$$
\delta_{j}=\sqrt{-\frac{c_{0}}{4 \lambda_{j} \alpha}+\frac{3}{4 h_{0}^{2}}-\frac{u_{0}}{2 \lambda_{j}}} \frac{\tanh \mu_{j}-r_{j}}{1-r_{j} \tanh \mu_{j}},
$$

where $r_{j}(0<j \leq 2 N)$ are nonzero constants.

Using the Cramer rule to solve the linear algebraic system (18), we obtain

$$
A_{N-1}=\frac{\Delta_{A_{N-1}}}{\Delta}
$$

where

$$
\begin{aligned}
\Delta & =\left|\begin{array}{cccccccccc}
1 & \delta_{1} & \lambda_{1} & \delta_{1} \lambda_{1} & \cdots & \lambda_{1}^{k} & \delta_{1} \lambda_{1}^{k} & \cdots & \lambda_{1}^{N-1} & \delta_{1} \lambda_{1}^{N-1} \\
1 & \delta_{2} & \lambda_{2} & \delta_{2} \lambda_{2} & \cdots & \lambda_{2}^{k} & \delta_{2} \lambda_{2}^{k} & \cdots & \lambda_{2}^{N-1} & \delta_{2} \lambda_{2}^{N-1} \\
\vdots & \vdots & \vdots & \vdots & \vdots & \vdots & \vdots & \vdots & \vdots & \vdots \\
1 & \delta_{2 N} & \lambda_{2 N} & \delta_{2 N} \lambda_{2 N} & \cdots & \lambda_{2 N}^{k} & \delta_{2 N} \lambda_{2 N}^{k} & \cdots & \lambda_{2 N}^{N-1} & \delta_{2 N} \lambda_{2 N}^{N-1}
\end{array}\right|, \\
\Delta_{A_{N-1}} & =\left|\begin{array}{cccccccccc}
1 & \delta_{1} & \lambda_{1} & \delta_{1} \lambda_{1} & \cdots & \lambda_{1}^{k} & \delta_{1} \lambda_{1}^{k} & \cdots & -\lambda_{1}^{N} & \delta_{1} \lambda_{1}^{N-1} \\
1 & \delta_{2} & \lambda_{2} & \delta_{2} \lambda_{2} & \cdots & \lambda_{2}^{k} & \delta_{2} \lambda_{2}^{k} & \cdots & -\lambda_{2}^{N} & \delta_{2} \lambda_{2}^{N-1} \\
\vdots & \vdots & \vdots & \vdots & \vdots & \vdots & \vdots & \vdots & \vdots & \vdots \\
1 & \delta_{2 N} & \lambda_{2 N} & \delta_{2 N} \lambda_{2 N} & \cdots & \lambda_{2 N}^{k} & \delta_{2 N} \lambda_{2 N}^{k} & \cdots & -\lambda_{2 N}^{N} & \delta_{2 N} \lambda_{2 N}^{N-1}
\end{array}\right| .
\end{aligned}
$$




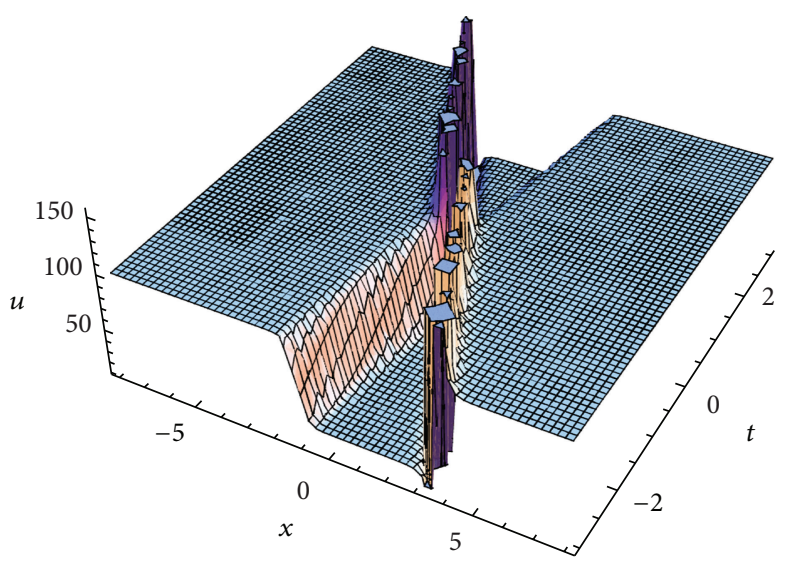

(a) $0.3<h_{0}<0.5, \lambda_{1}<0, \lambda_{2}<0$

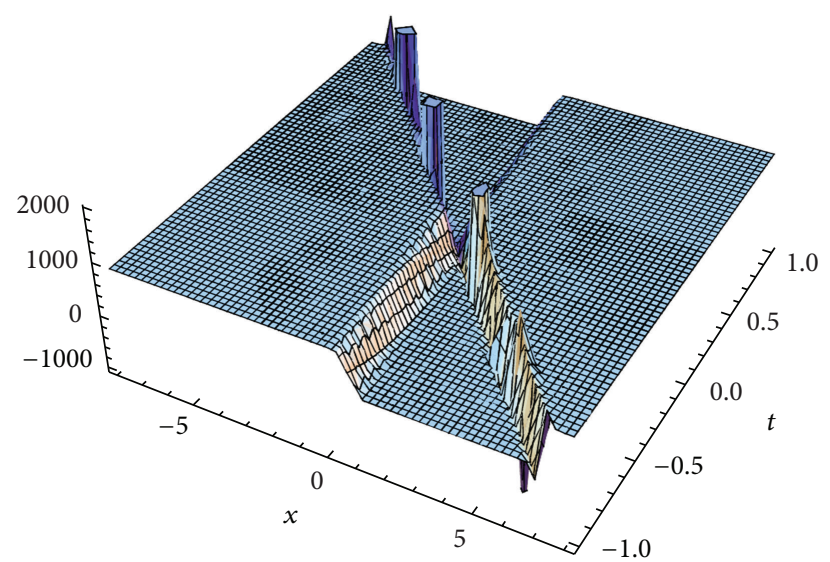

(b) $0.2<h_{0}<0.3, \lambda_{1}<0, \lambda_{2}<0$

FIGURE 1: The 3-D graphs of profiles of the singular double-soliton solution (53) for fixed parameters $c_{0}=1.5, \alpha=0.2, \lambda_{1}=-1, \lambda_{2}=-0.2$, $r_{1}=-8.0, r 2=2.0$, and $a=0.1$.

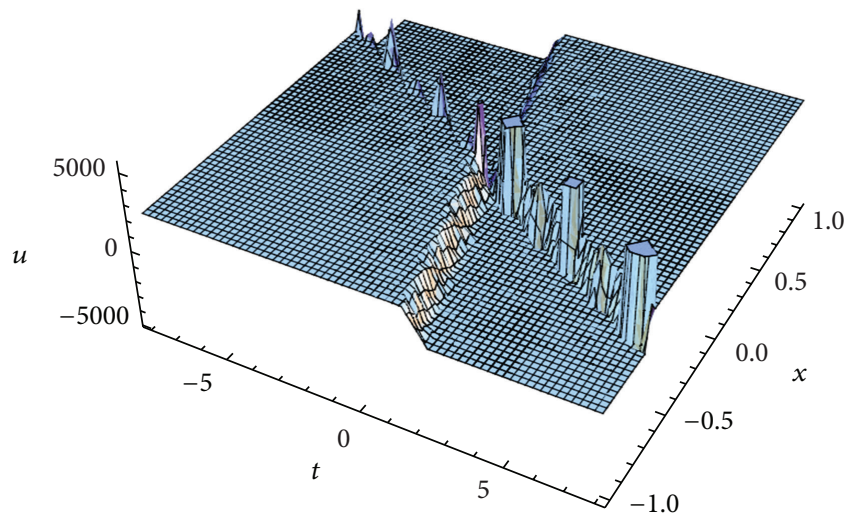

(a) $0<h_{0}<0.2, \lambda_{1}<0, \lambda_{2}<0$

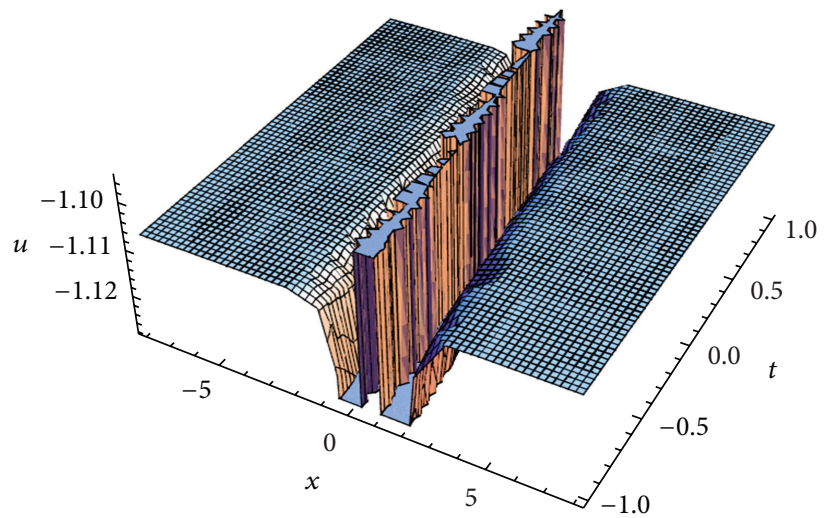

(b) $h_{0}>1, \lambda_{1}>0, \lambda_{2}>0$

FIGURE 2: The 3-D graphs of profiles of the singular double-soliton solution (53) for parameters: (a) $h_{0}=0.15, c_{0}=1.5, \alpha=0.2, \lambda_{1}=-1$, $\lambda_{2}=-0.2, r_{1}=-8.0, r_{2}=2.0$, and $a=0.1$; (b) $h_{0}=3, c_{0}=-1.5, \alpha=0.2, \lambda_{1}=1, \lambda_{2}=0.2, r_{1}=8, r_{2}=2.0$, and $a=-2$.

As examples, we will investigate exact solutions of (1) in two simple cases $N=1$ and $N=2$. When $N=1$, solving the linear algebraic system (18) leads to

$$
A_{0}=\frac{\delta_{1} \lambda_{2}-\delta_{2} \lambda_{1}}{\delta_{2}-\delta_{1}}
$$

Substituting (49) and (52) into (24), a singular double-soliton solution of (1) is obtained as follows:

$$
\begin{aligned}
\bar{u}[1]=\frac{6}{h_{0}^{2} f} & {\left[\sqrt{-\frac{c_{0}}{4 \lambda_{1} \alpha}+\frac{3}{4 h_{0}^{2}}-\frac{u_{0}}{2 \lambda_{1}}} \frac{\tanh \mu_{1}-r_{1}}{1-r_{1} \tanh \mu_{1}} \lambda_{2}\right.} \\
& \left.-\sqrt{-\frac{c_{0}}{4 \lambda_{2} \alpha}+\frac{3}{4 h_{0}^{2}}-\frac{u_{0}}{2 \lambda_{2}}} \frac{\tanh \mu_{2}-r_{2}}{1-r_{2} \tanh \mu_{2}} \lambda_{1}\right],
\end{aligned}
$$

where

$$
\begin{aligned}
f= & \sqrt{-\frac{c_{0}}{4 \lambda_{2} \alpha}+\frac{3}{4 h_{0}^{2}}-\frac{u_{0}}{2 \lambda_{2}}} \frac{\tanh \mu_{2}-r_{2}}{1-r_{2} \tanh \mu_{2}} \\
& -\sqrt{-\frac{c_{0}}{4 \lambda_{1} \alpha}+\frac{3}{4 h_{0}^{2}}-\frac{u_{0}}{2 \lambda_{1}}} \frac{\tanh \mu_{1}-r_{1}}{1-r_{1} \tanh \mu_{1}} .
\end{aligned}
$$

By using program of computer, it is easy to verify that the solution (53) satisfies (1), and this shows that the Darboux transformation (24) which we obtained is correct. In order to show the properties of the above singular double-soliton solutions visually, as an example, we plot the 3-D graphs of solution (53) for some fixed parameters, which are shown in Figures 1 and 2. 


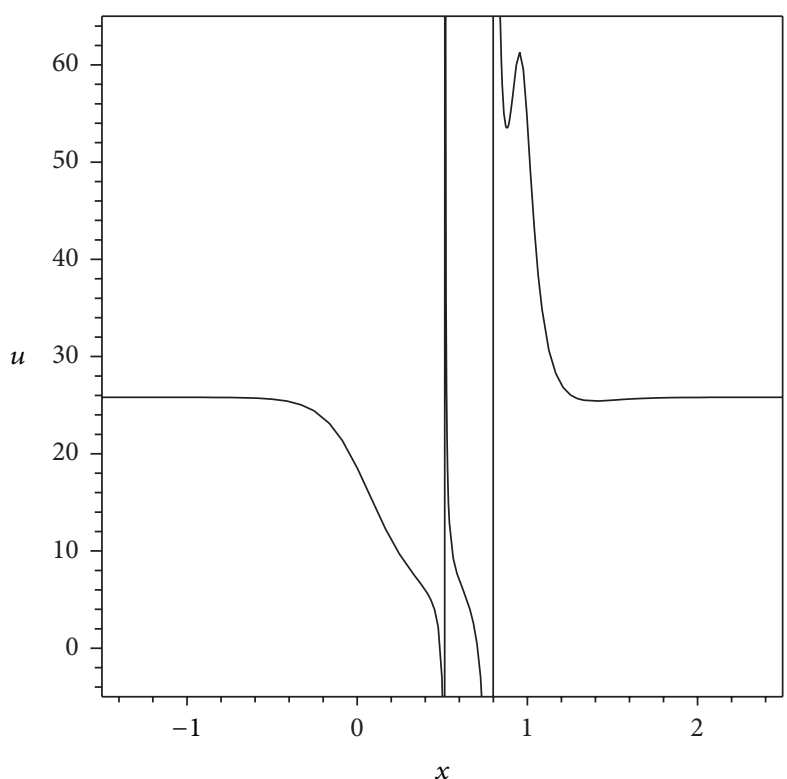

FIGURE 3: The 2-D graph of profile of the exact soliton solution (57) for fixed parameters.

When $N=2$, using the Cramer rule to solve the linear algebraic system (18), we obtain

$$
A_{1}=\frac{\Delta_{A_{1}}}{\Delta}
$$

with

$$
\begin{aligned}
\Delta & =\left|\begin{array}{llll}
1 & \delta_{1} & \lambda_{1} & \delta_{1} \lambda_{1} \\
1 & \delta_{2} & \lambda_{2} & \delta_{2} \lambda_{2} \\
1 & \delta_{3} & \lambda_{3} & \delta_{3} \lambda_{3} \\
1 & \delta_{4} & \lambda_{4} & \delta_{4} \lambda_{4}
\end{array}\right|, \\
\Delta_{A_{1}} & =\left|\begin{array}{llll}
1 & \delta_{1} & -\lambda_{1}^{2} & \delta_{1} \lambda_{1} \\
1 & \delta_{2} & -\lambda_{2}^{2} & \delta_{2} \lambda_{2} \\
1 & \delta_{3} & -\lambda_{3}^{2} & \delta_{3} \lambda_{3} \\
1 & \delta_{4} & -\lambda_{4}^{2} & \delta_{4} \lambda_{4}
\end{array}\right|,
\end{aligned}
$$

where $\delta_{j}(j=1,2,3,4)$ are given by (49). From (24), an explicit solution of (1) is obtained by the following:

$$
\bar{u}[2]=u+\frac{6}{h_{0}^{2}} A_{1}
$$

where $A_{1}$ is given by (55). Equation (57) is a very complex solution, and it is not soliton solution. In order to show the properties of solution (57), under the fixed parameters $\lambda_{1}=$ $-0.2, \lambda_{2}=-0.3, \lambda_{3}=-0.4, \lambda_{4}=-0.1, a=0, c_{0}=15, \alpha=1 / 2$, $h_{0}=0.8, r_{1}=-0.2, r_{2}=-0.3, r_{3}=-0.4, r_{4}=-0.5, t=0.1$, we plot its 2-D profile, which is shown in Figure 3.

\section{Acknowledgments}

This work is supported by the National Natural Science Foundation of China (no. 11361023), the Natural Science
Foundation of Chongqing Normal University (no. 13XLR20), the Scientific Foundation of Education of Yunnan Province (no. 2012C199), and the Program Foundation of Chongqing Innovation Team Project in University under Grant no. KJTD201308.

\section{References}

[1] J. Li, T. Xu, X. Meng, Y. Zhang, H. Zhang, and B. Tian, "Lax pair, Bäcklund transformation and $\mathrm{N}$-soliton-like solution for a variable-coefficient Gardner equation from nonlinear lattice, plasma physics and ocean dynamics with symbolic computation," Journal of Mathematical Analysis and Applications, vol. 336, no. 2, pp. 1443-1455, 2007.

[2] A. U. Khawaja, "A comparative analysis of Painlevé, Lax pair, and similarity transformation methods in obtaining the integrability conditions of nonlinear Schrödinger equations," Journal of Mathematical Physics, vol. 51, no. 5, Article ID 007005JMP, 2010.

[3] Q. X. Qu, B. Tian, K. Sun, and Y. Jiang, "Bäcklund transformation, Lax pair, and solutions for the Caudrey-Dodd-Gibbon equation," Journal of Mathematical Physics, vol. 52, no. 1, Article ID 013511, 2011.

[4] J. Lin, B. Ren, H. Li, and Y. Li, "Soliton solutions for two nonlinear partial differential equations using a Darboux transformation of the Lax pairs," Physical Review E, vol. 77, no. 3, Article ID 036605, 2008.

[5] P. Wang, B. Tian, W. Liu, Q. Qu, M. Li, and K. Sun, "Lax pair, conservation laws and $\mathrm{N}$-soliton solutions for the extended Korteweg-de Vries equations in fluids," European Physical Journal D, vol. 61, no. 3, pp. 701-708, 2011.

[6] Y. Jiang, B. Tian, W. Liu, M. Li, P. Wang, and K. Sun, "Solitons, Bäcklund transformation, and Lax pair for the $(2+1)$ dimensional Boiti-Leon-Pempinelli equation for the water waves," Journal of Mathematical Physics, vol. 51, no. 9, Article ID 093519, 2010.

[7] H. Zhi, "Symmetry reductions of the Lax pair for the $2+$ 1-dimensional Konopelchenko-Dubrovsky equation," Applied Mathematics and Computation, vol. 210, no. 2, pp. 530-535, 2009.

[8] E. Fan and K. W. Chow, "Darboux covariant Lax pairs and infinite conservation laws of the $(2+1)$-dimensional breaking soliton equation," Journal of Mathematical Physics, vol. 52, no. 2, Article ID 023504, 2011.

[9] H. Q. Zhang, B. Tian, T. Xu, H. Li, C. Zhang, and H. Zhang, "Lax pair and Darboux transformation for multi-component modified Korteweg-de Vries equations," Journal of Physics A, vol. 41, no. 35, Article ID 355210, 2008.

[10] X. Lü, B. Tian, K. Sun, and P. Wang, "Bell-polynomial manipulations on the Bäcklund transformations and Lax pairs for some soliton equations with one Tau-function," Journal of Mathematical Physics, vol. 51, no. 11, Article ID 113506, 2010.

[11] A. S. Fokas, "Lax pairs: a novel type of separability," Inverse Problems, vol. 25, no. 12, Article ID 123007, 2009.

[12] E. Fan, "New bilinear Bäcklund transformation and Lax pair for the supersymmetric two-Boson equation," Studies in Applied Mathematics, vol. 127, no. 3, pp. 284-301, 2011.

[13] S. F. Tian and H. Q. Zhang, "Lax pair, binary darboux transformation and new grammian solutions of nonisospectral kadomtsevpetviashvili equation with the two-singularmanifold method," Journal of Nonlinear Mathematical Physics, vol. 17, no. 4, pp. 491-502, 2010. 
[14] X. Lü, B. Tian, H. Q. Zhang, T. Xu, and H. Li, "Generalized (2+1)-dimensional Gardner model: bilinear equations, Bäcklund transformation, Lax representation and interaction mechanisms," Nonlinear Dynamics, vol. 67, no. 3, pp. 2279-2290, 2012.

[15] X. L. Gai, Y. T. Gao, L. Wang et al., "Painlevé property, Lax pair and Darboux transformation of the variable-coefficient modified Kortweg-de Vries model in fluid-filled elastic tubes," Communications in Nonlinear Science and Numerical Simulation, vol. 16, no. 4, pp. 1776-1782, 2011.

[16] J. Avan and A. Doikou, "Boundary Lax pairs for the An(1) Toda field theories," Nuclear Physics B, vol. 821, no. 3, pp. 481-505, 2009.

[17] Y. He and H. W. Tam, "Bilinear Bäcklund transformation and Lax pair for a coupled Ramani equation," Journal of Mathematical Analysis and Applications, vol. 357, no. 1, pp. 132136, 2009.

[18] H. Q. Zhang, B. Tian, X. H. Meng, X. Lü, and W. Liu, “Conservation laws, soliton solutions and modulational instability for the higher-order dispersive nonlinear Schrödinger equation," European Physical Journal B, vol. 72, no. 2, pp. 233-239, 2009.

[19] X. Yu, Y. T. Gao, Z. Y. Sun, and Y. Liu, "Wronskian solutions and integrability for a generalized variable-coefficient forced Korteweg-de Vries equation in fluids," Nonlinear Dynamics, vol. 67, no. 2, pp. 1023-1030, 2012.

[20] H. Q. Zhang, B. Tian, L. L. Li, and Y. Xue, "Darboux transformation and soliton solutions for the $(2+1)$-dimensional nonlinear Schrödinger hierarchy with symbolic computation," Physica A, vol. 388, no. 1, pp. 9-20, 2009.

[21] D. Levi, "On a new Darboux transformation for the construction of exact solutions of the Schrodinger equation," Inverse Problems, vol. 4, no. 1, pp. 165-172, 1988.

[22] V. B. Matveev and M. A. Salle, "Scattering of solitons in the formalism of the Darboux transform," Journal of Soviet Mathematics, vol. 34, no. 5, pp. 1983-1987, 1986.

[23] X. Geng and H. W. Tam, "Darboux transformation and soliton solutions for generalized nonlinear Schrödinger equations," Journal of the Physical Society of Japan, vol. 68, no. 5, pp. 15081512, 1999.

[24] Y. Li, W. X. Ma, and J. E. Zhang, "Darboux transformations of classical Boussinesq system and its new solutions," Physics Letters A, vol. 275, no. 1-2, pp. 60-66, 2000.

[25] X. Li and A. Chen, "Darboux transformation and multi-soliton solutions of Boussinesq-Burgers equation," Physics Letters A, vol. 342, no. 5-6, pp. 413-420, 2005.

[26] A. Chen and X. Li, "Darboux transformation and soliton solutions for Boussinesq-Burgers equation," Chaos, Solitons and Fractals, vol. 27, no. 1, pp. 43-49, 2006.

[27] D. Levi, "On a new Darboux transformation for the construction of exact solutions of the Schrodinger equation," Inverse Problems, vol. 4, no. 1, pp. 165-172, 1988.

[28] Z. B. Zhaqilao and Z. Li, "Darboux transformation and bidirectional soliton solutions of a new $(2+1)$-dimensional soliton equation," Physics Letters A, vol. 372, no. 9, pp. 1422-1428, 2008.

[29] H. X. Yang, "Soliton solutions by Darboux transformation for a Hamiltonian lattice system," Physics Letters A, vol. 373, no. 7, pp. 741-748, 2009.

[30] X. Wu, W. Rui, and X. Hong, "A new discrete integrable system derived from a generalized Ablowitz-Ladik hierarchy and its Darboux transformation," Discrete Dynamics in Nature and Society, vol. 2012, Article ID 652076, 19 pages, 2012.
[31] W. X. Ma, "Darboux transformations for a lax integrable system in 2n dimensions," Letters in Mathematical Physics, vol. 39, no. 1, pp. 33-49, 1997.

[32] W. X. Ma, "The algebraic structure of zero curvature representations and application to coupled KdV systems," Journal of Physics A, vol. 26, no. 11, pp. 2573-2582, 1993.

[33] R. Camassa and D. D. Holm, "An integrable shallow water equation with peaked solitons," Physical Review Letters, vol. 71, no. 11, pp. 1661-1664, 1993.

[34] G. B. Whitham, Linear and Nonlinear Waves, Wiley, New York, NY, USA, 1974.

[35] B. Fuchssteiner and A. S. Fokas, "Symplectic structures, their Bäcklund transformations and hereditary symmetries," Physica D, vol. 4, no. 1, pp. 47-66, 1981.

[36] B. Fuchssteiner, "The Lie algebra structure of nonlinear evolution equations admitting infinite dimensional abelian symmetry groups," Progress of Theoretical Physics, vol. 65, no. 3, pp. 861876, 1981.

[37] A. S. Fokas and P. M. Santini, "An inverse acoustic problem and linearization of moderate amplitude dispersive waves," 1994.

[38] A. S. Fokas, "On a class of physically important integrable equations," Physica D, vol. 87, no. 1-4, pp. 145-150, 1995.

[39] Q. Bi, "Wave patterns associated with a singular line for a biHamiltonian system," Physics Letters A, vol. 369, no. 5-6, pp. 407-417, 2007.

[40] J. Li and J. Zhang, "Bifurcations of travelling wave solutions for the generalization form of the modified KdV equation," Chaos, Solitons and Fractals, vol. 21, no. 4, pp. 899-913, 2004.

[41] W. Rui, B. He, Y. Long, and C. Chen, “The integral bifurcation method and its application for solving a family of third-order dispersive PDEs," Nonlinear Analysis, Theory, Methods and Applications, vol. 69, no. 4, pp. 1256-1267, 2008.

[42] W. Rui, C. Chen, X. Yang, and Y. Long, "Some new solitonlike solutions and periodic wave solutions with loop or without loop to a generalized KdV equation," Applied Mathematics and Computation, vol. 217, no. 4, pp. 1666-1677, 2010.

[43] Y. Long and C. Chen, "Existence analysis of traveling wave solutions for a generalization of $\mathrm{KdV}$ equation," Mathematical Problems in Engineering, vol. 2013, Article ID 462957, 7 pages, 2013. 


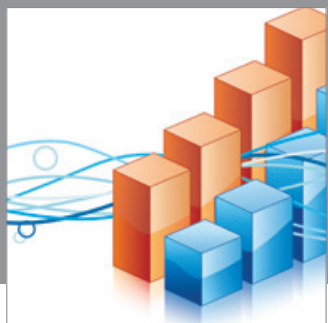

Advances in

Operations Research

mansans

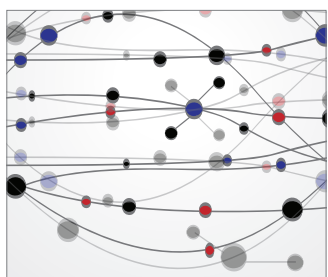

The Scientific World Journal
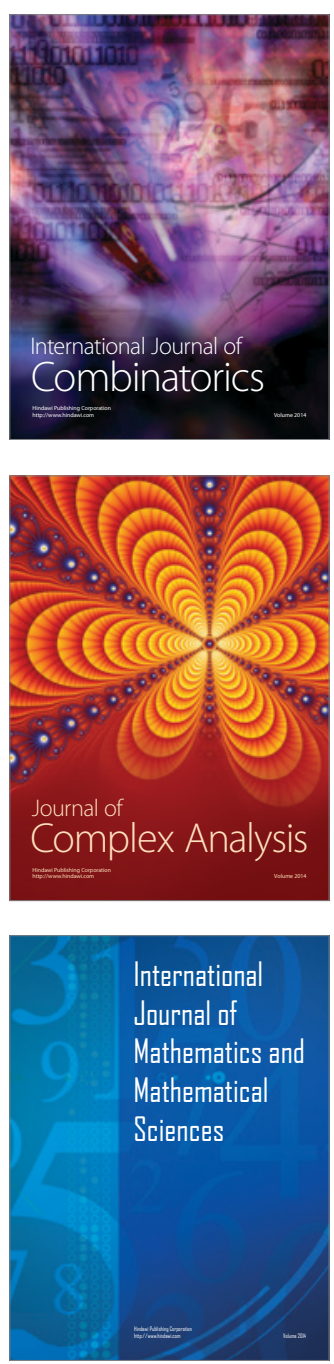
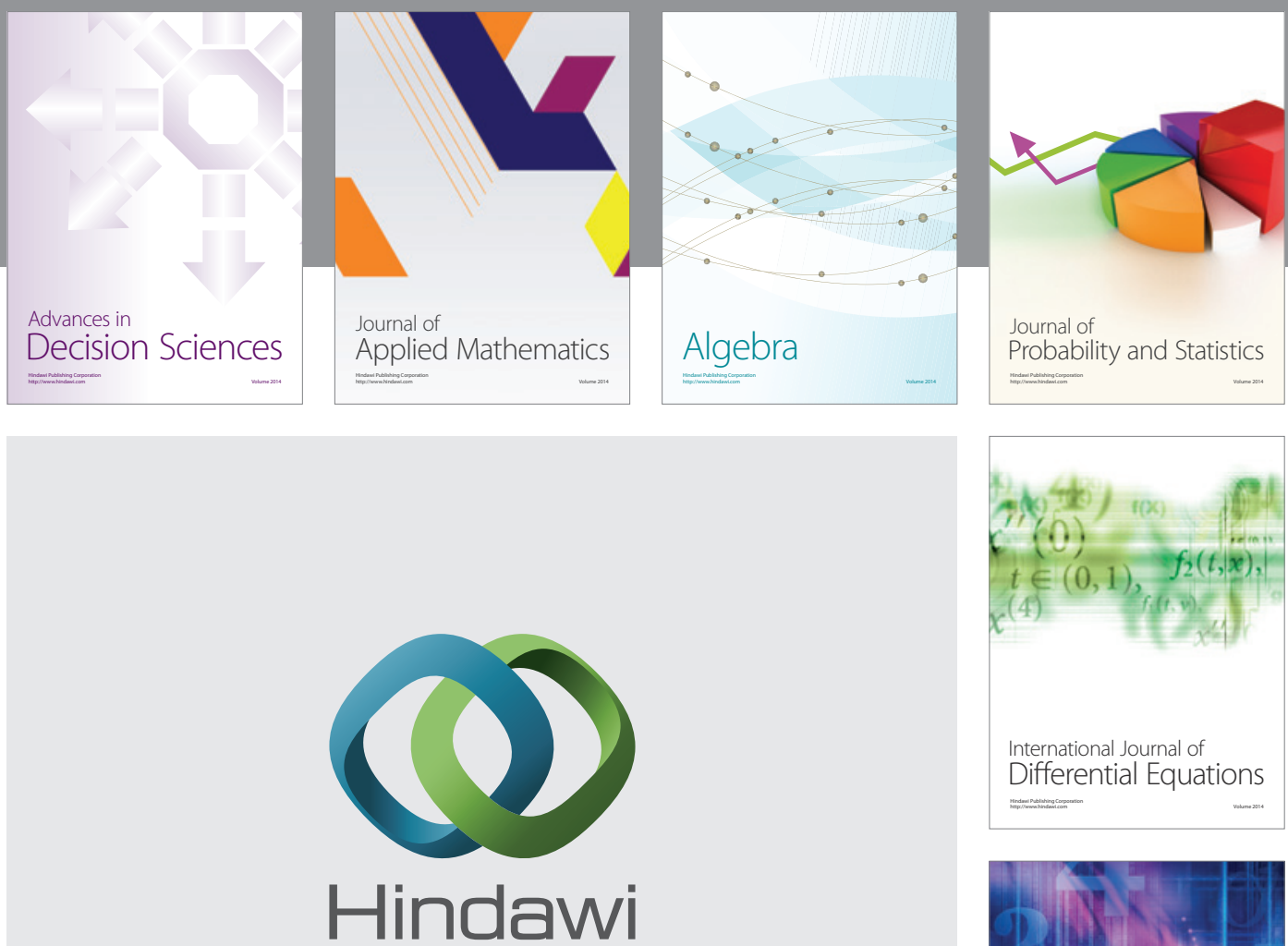

Submit your manuscripts at http://www.hindawi.com
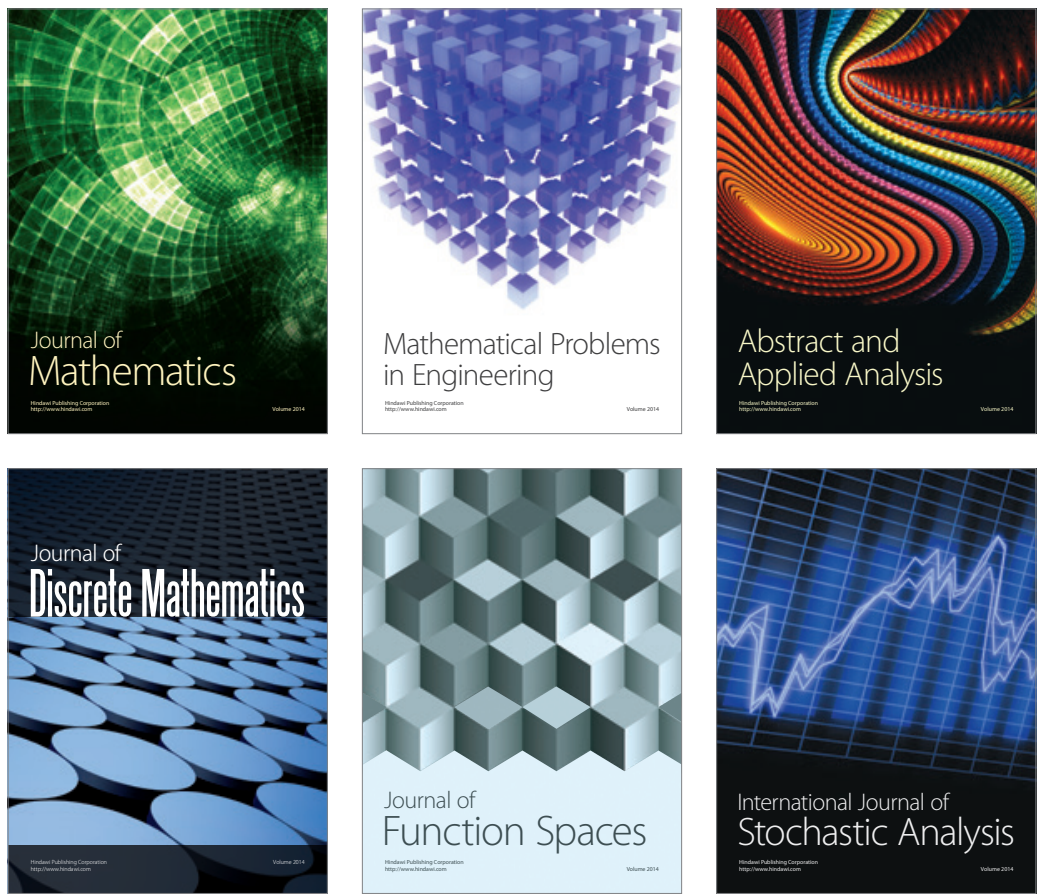

Journal of

Function Spaces

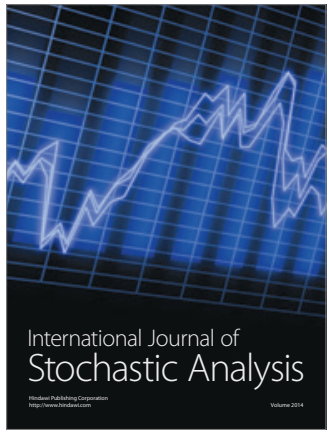

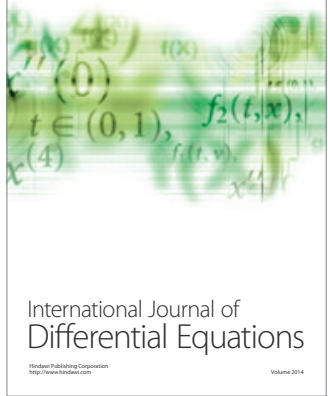
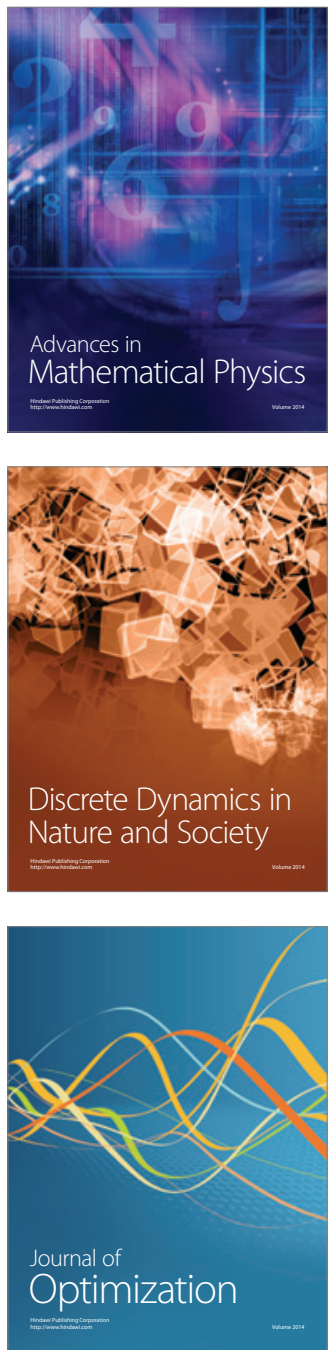